\title{
Investigation on the Band Gap and Negative Properties of Concentric Ring Acoustic Metamaterial
}

\author{
Meng Chen, ${ }^{1,2}$ Dan Meng, ${ }^{1,2}$ Heng Jiang $\mathbb{D},^{1,2}$ and Yuren Wang $\mathbb{D}$ 1,2 \\ ${ }^{1}$ Key Laboratory of Microgravity, Institute of Mechanics, Chinese Academy of Sciences, Beijing 100190, China \\ ${ }^{2}$ University of Chinese Academy of Sciences, Beijing 100049, China \\ Correspondence should be addressed to Heng Jiang; hengjiang@imech.ac.cn and Yuren Wang; yurenwang@imech.ac.cn
}

Received 20 July 2017; Revised 4 December 2017; Accepted 24 December 2017; Published 24 January 2018

Academic Editor: Marcello Vanali

Copyright (c) 2018 Meng Chen et al. This is an open access article distributed under the Creative Commons Attribution License, which permits unrestricted use, distribution, and reproduction in any medium, provided the original work is properly cited.

\begin{abstract}
The acoustic characteristics of 2D single-oscillator, dual-oscillator, and triple-oscillator acoustic metamaterials were investigated based on concentric ring structures using the finite element method. For the single-oscillator, dual-oscillator, and triple-oscillator models investigated here, the dipolar resonances of the scatterer always induce negative effective mass density, preventing waves from propagating in the structure, thus forming the band gap. As the number of oscillators increases, relative movements between the oscillators generate coupling effect; this increases the number of dipolar resonance modes, causes negative effective mass density in more frequency ranges, and increases the number of band gaps. It can be seen that the number of oscillators in the cell is closely related to the number of band gaps due to the coupling effect, when the filling rate is of a certain value.
\end{abstract}

\section{Introduction}

Acoustic metamaterials possess certain peculiar acoustic characteristics that natural materials do not have (e.g., negative effective mass density, negative effective modulus) and as such have garnered considerable attention for their promise in applications such as acoustic/elastic filters, acoustic mirrors, and sound insulators/absorbers $[1,2]$. Acoustic metamaterials are usually composed of a series of periodically arranged resonance units [3]. A negative effective mass density is generated by dipolar resonance at low frequencies [4-6], while a negative effective bulk modulus is caused by monopolar resonance at low frequencies [79]. Monopolar and dipolar resonance unit coupling can simultaneously achieve negative effective mass density and negative effective bulk modulus; thus, to achieve the most favorable possible acoustic properties, researchers often focus on designing the unit structures rather than altering material compositions [10-12]. Previous researchers have established structural units for reference when building new acoustic metamaterials and acoustic devices; however, most of the literatures are centered on single-oscillator systems, which leave the acoustic metamaterials with fewer regulation factors
[13]. The multioscillator system, conversely, allows a plurality of mutually coupled oscillators within one unit $[13$, 14]. As opposed to the single-oscillator system, it contains multiple vibration modes and more degrees of freedom can be regulated. Interestingly, however, relatively few studies have investigated its specific structure or unique acoustic properties. In this study, a multioscillator system examined the acoustic properties by introducing a metamaterial system with concentric ring structure.

The multioscillator system here investigated was created by introducing multiple resonators into a unit cell and generating new forms of resonance through mutual coupling between each oscillator in order to control acoustic properties $[13,14]$. Previous studies have shown that compared to single-oscillator systems multioscillator acoustic metamaterial systems readily facilitate wider bandwidth [13]. However, due to the complex structure of the multioscillator acoustic metamaterial system, it is a rather challenging endeavor to fully define the formation mechanism of its acoustic properties. The concentric ring structure in our test system was designed by introducing a series of coaxial resonators (i.e., a typical multioscillator system). Its structure is relatively simple and it is characterized by strong links between the 

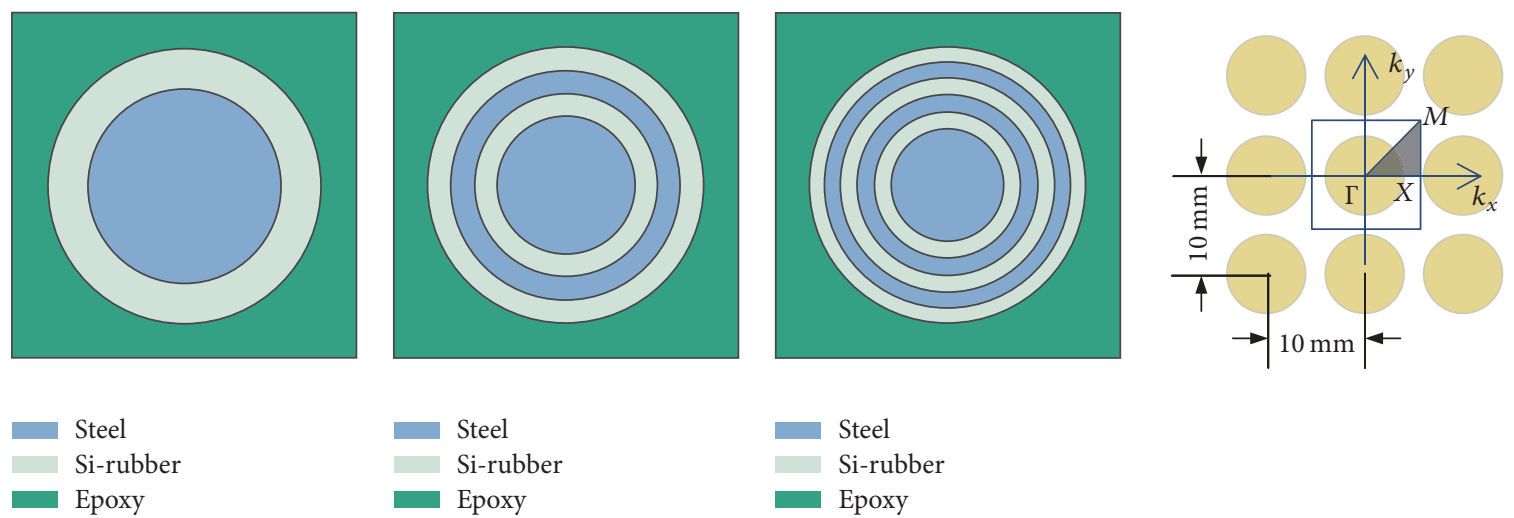

(a)

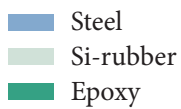

(b)

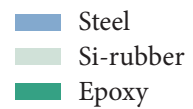

(c) (d)

Figure 1: Cross-sectional views of 2D acoustic metamaterials in single-oscillator, dual-oscillator, and triple-oscillator systems (a-c); units in square lattice arrangement (lattice constant $=10 \mathrm{~mm}$ ) and schematic diagram of the first Brillouin zone $(\mathrm{d})$. Note. Shaded area is the irreducible Brillouin zone. Points $\Gamma, X$, and $M$ are irreducible Brillouin vertexes.

oscillators. The number of oscillators can be freely controlled by adjusting the number of scatterer layers. This structure, as discussed in detail below, proved ideal for investigating the acoustic properties of the multioscillator system.

A sequence of concentric circular structures with alternating properties used as Bragg fiber was widely investigated in photonic crystal $[15,16]$, and recently researchers have conducted preliminary studies on acoustic metamaterials arranged in concentric rings, which can be used as sound filter. For example, Larabi et al. studied the concentric ring structure of a system with water as the matrix, focusing on the manner in which transmission response varies with the number of oscillators, and found that in the transmission response curve, the number of attenuation peaks increases as the number of oscillators increases [17]. Torrent and Sánchezdehesa demonstrated the acoustic metamaterials with the radially periodic structure and developed a new class of structures named radial wave crystals [18]. Subsequently, the low frequency band structures of twodimensional (2D) arc-shaped phononic crystals were studied by utilizing the transfer-matrix method [19]. Zhou and Chen introduced electrorheological (ER) fluids in concentric rings (considered as a soft layer in the system) and proved that the dual-oscillator structure has more band gaps than the single-oscillator structure [20]. Basically, the concentric ring structure is a typical multioscillator system, the acoustic properties of which are enhanced by larger quantities of oscillators. Studies on this subject have focused primarily on the transmission characteristics or band gaps while neglecting the effective medium parameters. In effort to remedy this, the acoustic characteristics of our test concentric ring structure were investigated via numerical calculation. As discussed below, the formation mechanism and rules for the acoustic properties in the multioscillator system were ultimately identified.

The concentric ring structures were designed to build 2D single-oscillator, dual-oscillator, and triple-oscillator acoustic metamaterial systems and the properties of each were thoroughly analyzed by numerically analyzing and comparing their respective band structures, transmission characteristics, effective medium parameters, and other necessary factors. It was found that as the number of oscillators increases the number of vibrational degrees of freedom of the system increases. The interaction between the oscillators at a plurality of frequencies caused negative effective mass density while the modulus remained positive. According to the effective medium theory, the waves at these frequencies were not able to propagate; thus the number of acoustic/elastic wave bands increased significantly.

\section{Models and Calculation Methods}

Concentric ring structures can be introduced into a $2 \mathrm{D}$ acoustic metamaterial to build a multioscillator system. In this study, the unit section is designed as a set of concentric rings with alternating hard and soft material, and the number of oscillators in the entire system is adjusted by changing the number of layers of concentric rings. As mentioned above, a single-oscillator, dual-oscillator, and triple-oscillator system are built. A unit sectional view of the single-oscillator acoustic metamaterial system is shown in Figure 1(a), where the scatterer is comprised of infinitely long steel (cylindrical) columns evenly coated with Si-rubber; Figure 1(b) shows a unit sectional view of the dual-oscillator acoustic metamaterial system, where the interior contains two layers of alternately arranged, concentrically coated steel columns; and Figure 1(c) shows a unit sectional view of the triple-oscillator acoustic metamaterial system, where the interior contains three layers of alternately arranged, concentrically coated steel columns.

Previous studies have demonstrated that the filling factor impacts the acoustic characteristics of acoustic metamaterial systems [21]. To eliminate the influence of filling factor, the radii of the three scatterers are set to $4 \mathrm{~mm}$ and each was embedded in an epoxy matrix on a square lattice with a lattice parameter of $10 \mathrm{~mm}$ such that the filling factor was $50 \%$. The scatterers are all constituted by steel and Si-rubber at $1: 1$ ratio. Previous studies also typically simplify this type of system 
TABLE 1: Scatterer dimensions.

\begin{tabular}{lcccccc}
\hline & $R_{1}(\mathrm{~mm})$ & $R_{2}(\mathrm{~mm})$ & $R_{3}(\mathrm{~mm})$ & $R_{4}(\mathrm{~mm})$ & $R_{5}(\mathrm{~mm})$ & -- \\
\hline Single-oscillator & 2.83 & 4.00 & -- & -- & -- \\
Dual-oscillator & 2.00 & 2.67 & 3.33 & 4.00 & -- & -- \\
Triple-oscillator & 1.63 & 2.09 & 2.65 & 3.11 & 3.54 & 4.00 \\
\hline
\end{tabular}

Note. $R_{1}$ to $R_{6}$ are the radii from inner to outer layers of scatters.

TABLE 2: Mass densities and elastic constants of the materials.

\begin{tabular}{lccc}
\hline Materials & Epoxy & Si-rubber & Steel \\
\hline Mass density, $\rho\left(\mathrm{kg} / \mathrm{m}^{3}\right)$ & 1180 & 1300 & 7780 \\
Young's modulus, $E(\mathrm{MPa})$ & $0.435 \times 10^{4}$ & $1.175 \times 10^{-1}$ & $21.06 \times 10^{4}$ \\
Shear modulus, $\mu(\mathrm{MPa})$ & $0.159 \times 10^{4}$ & $4 \times 10^{-2}$ & $8.1 \times 10^{4}$ \\
\hline
\end{tabular}

into a mass-spring mode. It is generally accepted that the mass of model oscillators is mainly related to the volume of each steel layer and that the stiffness of springs is mainly related to the thickness of the soft coating layer and the contact areas [22]. To ensure that the masses of oscillators were uniformly distributed within the system, each hard layer is designed in the dual- and triple-oscillator systems to have similar volume. As such, each soft layer had uniform thickness, so the spring stiffness does not vary significantly throughout the system. The sizes of each element are listed in Table 1 and the physical parameters of the material components are listed in Table 2.

Effective medium parameters, in addition to band structure, transmission response, and other factors related to acoustic properties, require definition [23-26]. In this study, the effective medium theory is applied to analyze wave propagation through materials in our test systems. Theoretical calculations of acoustic metamaterials behavior mainly use multiple-scattering (MS) methods, plane-wave-expansion (PWE) methods, the finite-difference time-domain (FDTD) method, transfer-matrix methods, lumped-mass methods, and finite element methods [27-33]. Finite element methods (FEMs) are commonly used to calculate the band structure and vibration modes of LRSMs $[34,35]$. A distinct advantage of the FEM is the flexibility of modeling various materials with complex structure, good convergence, and high precision [35]. So the FEM software COMSOL Multiphysics is used to calculate the band structure, transmission response, and effective medium parameters (i.e., effective mass density, effective bulk modulus, and effective shear modulus) of the single-oscillator, dual-oscillator, and triple-oscillator concentric ring acoustic metamaterial systems.

The calculation model of the band structure can be reduced to a single unit by applying the Bloch boundary on the two opposite boundaries [33]. The whole band structure can be obtained by letting the wave vector sweep the edges of the irreducible Brillouin zone, which is marked by the shaded region in Figure $1(\mathrm{~d})$. The transmission response can be calculated using the finite periodic model, which is defined according to only four units along the wave propagation direction. The computing methods described in previous investigations are referred to for calculating the effective medium parameters [36]. In calculations, the triangle element mesh is used. It is worth noting that, in order to ensure the accuracy of the calculation, the maximum element size of the triangle mesh is far less than $1 / 6$ of the shortest wavelength in calculated rage of frequencies.

\section{Results and Discussion}

3.1. Single-Oscillator Acoustic Metamaterial System. The band structure of the single-oscillator acoustic metamaterial system in the $\Gamma X$ direction is shown in Figure 2(a). There are two band gaps and the ranges of them are $846 \mathrm{~Hz}-1542 \mathrm{~Hz}$ and $2571 \mathrm{~Hz}-2915 \mathrm{~Hz}$. Figure 3 shows the vibration modes at the first band gap edges. At the lower edge, steel columns move and the matrix remains almost completely still, where the coating layer connects the steel column acting as a spring. At the upper edge, the matrix and steel columns move along opposite directions and the coating layer still acts as a spring. For a single-oscillator acoustic metamaterial system, the simplified mass-spring model can be used to estimate the frequencies on lower and upper edges of the first band gap (resp., $f_{L}$ and $f_{U}$ ) $[22,37]$ :

$$
\begin{gathered}
f_{L}=\frac{1}{2 \pi} \sqrt{\frac{k}{m_{1}}}, \\
f_{U}=\frac{1}{2 \pi} \sqrt{\frac{k\left(m_{1}+m_{2}\right)}{m_{1} m_{2}}},
\end{gathered}
$$

where $k$ is the stiffness of the simplified mass-spring model and $m_{1}$ and $m_{2}$ are the masses in the simplified model. Our calculations show that $f_{L}=839.79 \mathrm{~Hz}$ and $f_{U}=1549.15 \mathrm{~Hz}$. The band gap at the highest frequency range is narrower for those three systems, so the corresponding vibration modes are not discussed in this paper.

Sound waves cannot propagate in the material in the band gap frequency range. In order to verify the band structure, the transmission characteristics are examined via finite element method. The transmission characteristics for the longitudinal or transverse waves are then obtained by calculating the ratio of integrating the horizontal or vertical displacements on date line (the calculation methods are shown in the Appendix). Figure 2(b) shows the transmission characteristic curve of the single-oscillator acoustic metamaterial system in the $\Gamma X$ direction, where the blue solid and green dashed lines 


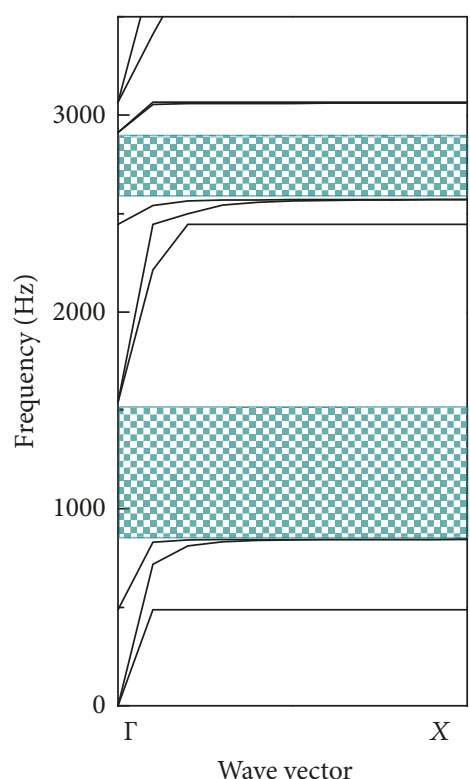

Wave vector

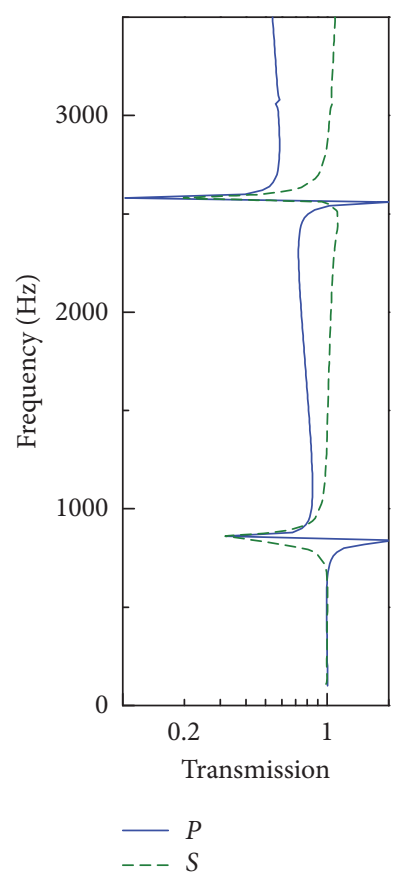

(b)

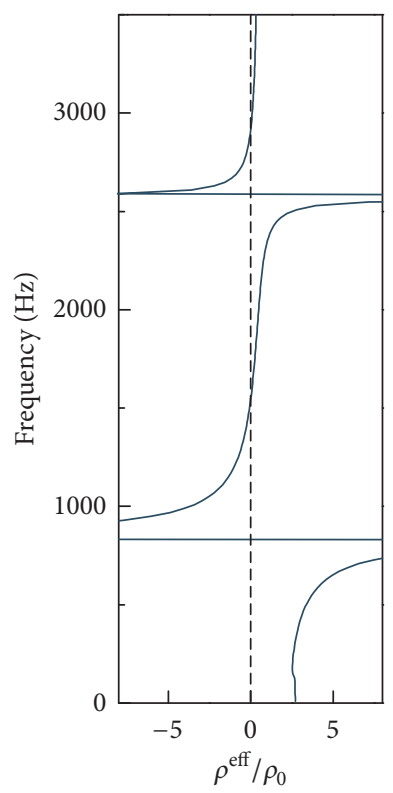

(c)

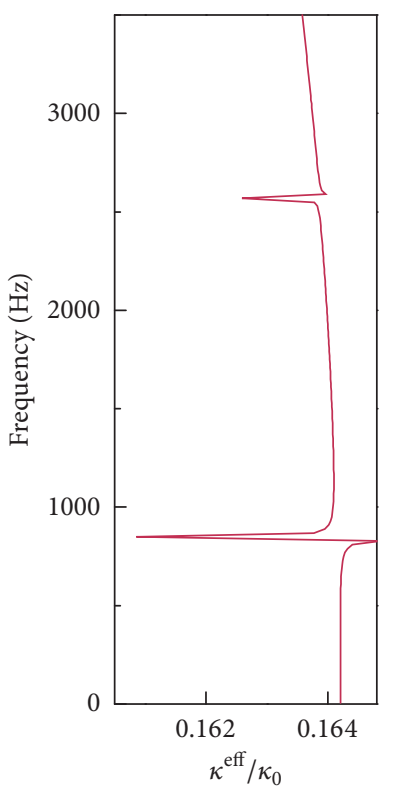

(d)

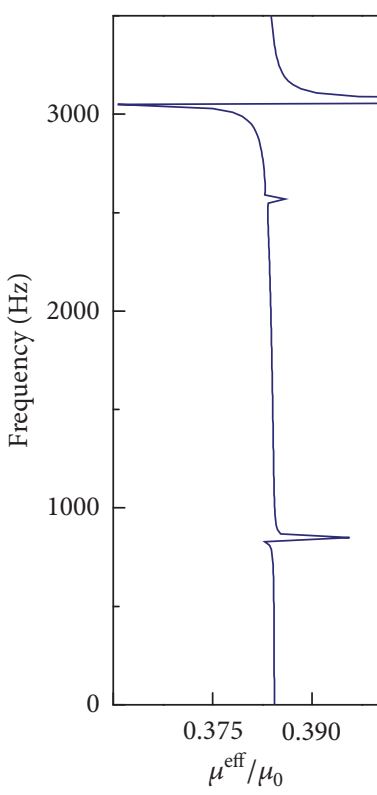

(e)

FIGURE 2: Band structure of single-oscillator acoustic metamaterial in the $\Gamma X$ direction (a); transmission characteristic curve in the $\Gamma X$ direction (b); normalized effective mass density, effective bulk modulus, and effective shear modulus (c-e). The blue regions represent the positions of band gaps. $P$ and $S$ represent a compression wave ( $p$-wave) and shear wave ( $s$-wave), respectively.

represent a compression wave ( $p$-wave) and shear wave $(s$ wave), respectively. The maximum vibration damping values of the $p$-wave and $s$-wave appeared at $860 \mathrm{~Hz}$ and $2580 \mathrm{~Hz}$, respectively. These frequencies correspond to the original frequencies of the band gaps (there were some deviations worth mentioning, however; actually, the transmission dip is generally different from the lower band gap edge because the transmission is attenuated when evanescent waves are generated $[38,39])$. In other words, these frequency ranges are the acoustic band gaps. With the number of unit cells increasing, the transmission gaps tend to be larger and deeper, but few units are sufficient to indicate the frequencies of band gaps [40]. The curves for both the $p$-wave and $s$-wave also show Fano-like interference phenomena [41]; that is, within some specific frequency ranges, highly asymmetrical peaks appeared in the transmission curve. 


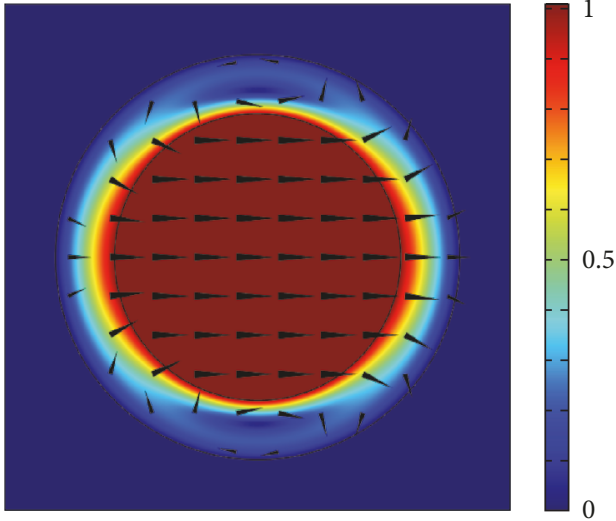

(a)

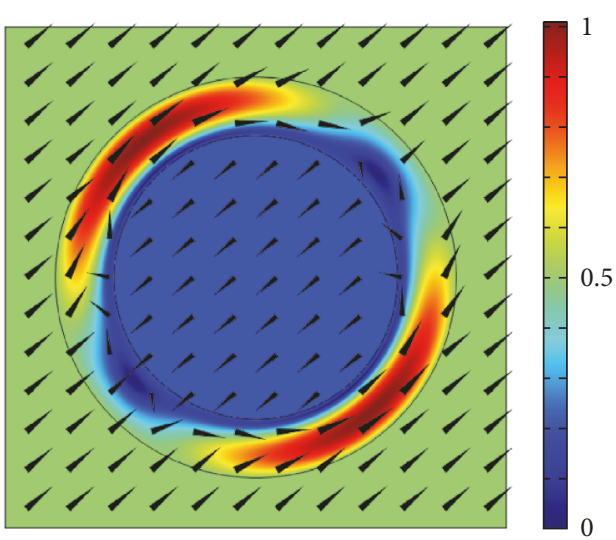

(b)

Figure 3: Vibration modes at lower edge (a) and upper edge (b) of the first band gap of the single-oscillator acoustic metamaterial system. The color bar represents the normalized displacement.

With a lattice constant of about $1 \mathrm{~cm}$, the shortest wavelength of compression wave and shear wave is about 15 times larger than that of the lattice constant in the frequency band of $0-10000 \mathrm{~Hz}$. From the effective medium theories, wave behaviors are described by effective parameters that may be obtained using surface integration method [36]. Figures $2(\mathrm{c})-2(\mathrm{e})$ show the normalized effective mass density $\rho^{\mathrm{eff}} / \rho_{0}$, effective bulk modulus $\kappa^{\text {eff }} / \kappa_{0}$, and effective shear modulus $\mu^{\text {eff }} / \mu_{0}$ of the single-oscillator acoustic metamaterial, where $\rho_{0}, \kappa_{0}$, and $\mu_{0}$ are the mass density, bulk modulus, and shear modulus of the matrix, respectively. In Figure $2(\mathrm{c}), \rho^{\text {eff }} / \rho_{0}$ become negative at $849 \mathrm{~Hz}-1529 \mathrm{~Hz}$ and $2589 \mathrm{~Hz}-2909 \mathrm{~Hz}$, respectively. Compared to the band structure shown in Figure 2(a), it is clear that the two negative $\rho^{\text {eff }} / \rho_{0}$ frequency ranges correspond to the first band gap and the second band gap; $\rho^{\text {eff }} / \rho_{0}$ first increases rapidly and then suddenly drops to a negative value near the original frequency of the band gaps and then gradually increases and becomes positive near the frequencies on upper edge of the band gaps. In the vibration modes, the negative effective mass density in the system formed mainly due to the dipolar resonances of the unit. Moreover, both $\kappa^{\text {eff }} / \kappa_{0}$ and $\mu^{\text {eff }} / \mu_{0}$ are positive within the frequency range of $0 \mathrm{~Hz}-3500 \mathrm{~Hz}$; thus the singleoscillator acoustic metamaterials are single negative acoustic metamaterials.

3.2. Dual-Oscillator Acoustic Metamaterials. Figure 4(a) shows the band structure of the dual-oscillator acoustic metamaterial system in the $\Gamma X$ direction. Band gaps form in the $946 \mathrm{~Hz}-1562 \mathrm{~Hz}, 2283 \mathrm{~Hz}-2835 \mathrm{~Hz}$, and $4247 \mathrm{~Hz}-4465 \mathrm{~Hz}$ frequency ranges. Figures 5(a) and 5(b) show the vibration modes at the lower edge and upper edge of the first band gap. In this band gap, the vibration mode at the lower edge is where two steel columns vibrate in the same direction while the matrix does not move and the energy was concentrated on the central steel columns; the vibration mode at the upper edge is where the matrix and the outer steel columns move opposite the central steel columns and the energy is concentrated in the matrix and central steel column. Similar to the vibration mode of the first band gap, Figures 5(c) and 5(d) show the vibration modes at edges of the second band gap. In this band gap, at the lower edge, the two steel columns vibrate opposite each other while the matrix does not move and the energy is concentrated on the outer steel column; at the upper edge, the matrix and the central steel column vibrate opposite the outer steel column and the energy is concentrated on the outer steel column and the matrix.

During vibration in the dual-oscillator acoustic metamaterial system, each layer of steel columns moves as a whole independently of other layers and the Si-rubber layer connects the matrix and each steel column like a spring. The vibration modes at the lower edges of the band gaps are controlled only by the scatterer (the matrix does not move). Accordingly, the lower edges of band gaps are evaluated by calculating the eigenfrequencies of a simplified mass-spring model which include two mass blocks and two springs. A similar method is used as a previous study to evaluate the masses of mass blocks and the stiffness of springs [22]. The evaluated values of the lower edges of the first and the second band gaps are $948 \mathrm{~Hz}$ and $2868 \mathrm{~Hz}$, respectively, and the results are close to the numerical solutions.

Figure 4(b) shows the transmission characteristic curves of the dual-oscillator acoustic metamaterial system in the $\Gamma X$ direction, where the blue solid line and green dashed line represent the $p$-wave and $s$-wave, respectively. The maximum values of $p$-wave and $s$-wave vibration damping appear at $970 \mathrm{~Hz}, 2290 \mathrm{~Hz}$, and $4260 \mathrm{~Hz}$. The maximum vibration damping values correspond to the original frequencies of the band gaps, and a Fano-like interference phenomenon also appears.

As shown in Figure $4(\mathrm{c}), \rho^{\mathrm{eff}} / \rho_{0}$ becomes negative at $986 \mathrm{~Hz}-1561 \mathrm{~Hz}, 2286 \mathrm{~Hz}-2811 \mathrm{~Hz}$, and $4261 \mathrm{~Hz}-4461 \mathrm{~Hz}$ and changes in a similar manner to those in the single-oscillator system. In the $0 \mathrm{~Hz}-5000 \mathrm{~Hz}$ frequency range, $\kappa^{\text {eff }} / \kappa_{0}$ and $\mu^{\text {eff }} / \mu_{0}$ are positive; therefore, the dual-oscillator acoustic metamaterials are single negative acoustic metamaterials. As evidenced by the vibration modes we observed, although 

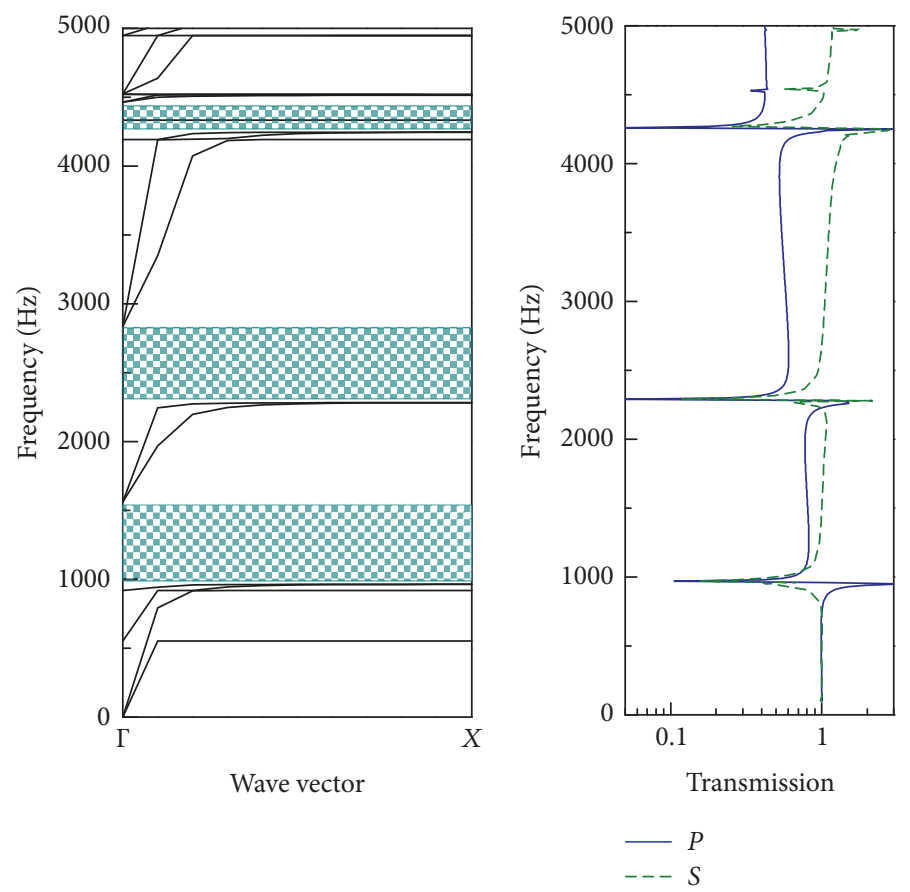

(a)

(b)

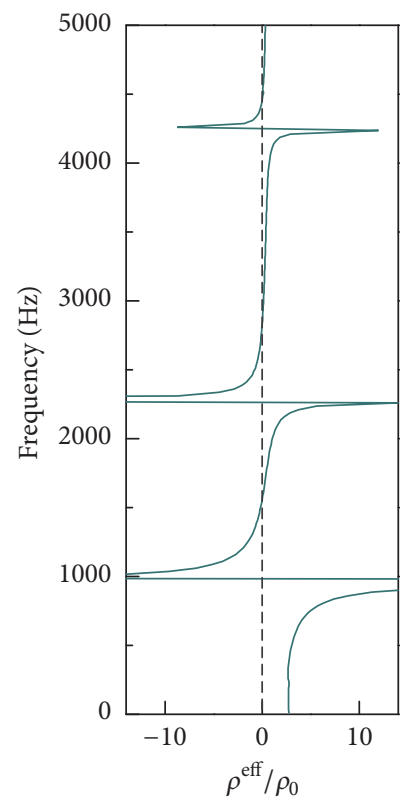

(c)

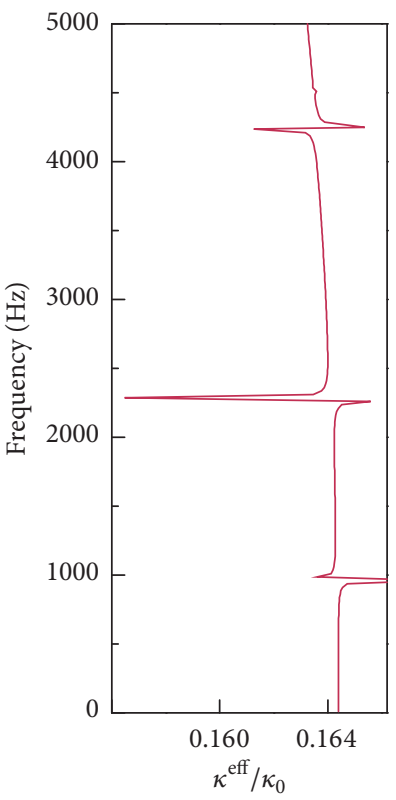

(d)

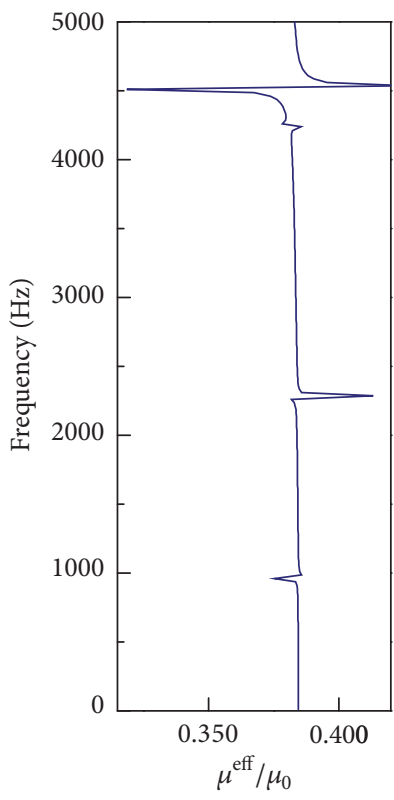

(e)

FIGURE 4: Band structure of dual-oscillator acoustic metamaterial in the $\Gamma X$ direction (a); transmission characteristic curve in the $\Gamma X$ direction (b); normalized effective mass density, effective bulk modulus, and effective shear modulus (c-e).

each layer of the scatterer has a relative motion, the vibrations still contain dipolar resonance. To this effect, the vibration of the scatterer of the dual-oscillator system induces negative effective mass density and a positive modulus.

3.3. Triple-Oscillator Acoustic Metamaterials. Figure 6(a) shows the band structure of the triple-oscillator acoustic metamaterial system in the $\Gamma X$ direction. Band gaps form in the $1021 \mathrm{~Hz}-1621 \mathrm{~Hz}, 2452 \mathrm{~Hz}-2956 \mathrm{~Hz}, 3773 \mathrm{~Hz}-4125 \mathrm{~Hz}$, and $6103 \mathrm{~Hz}-6257 \mathrm{~Hz}$. Figures 7(a) and 7(b) show the vibration modes at the lower edge and upper edge of the first band gap. In this band gap, at the lower edge, the three steel columns vibrate in the same direction while the matrix did not move and the energy is concentrated on the central steel column; at the upper edge, the central steel column and intermediate steel column move opposite the matrix and the 


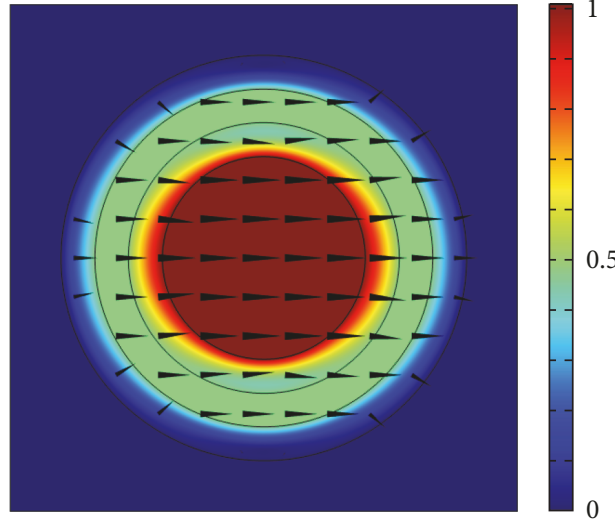

(a)

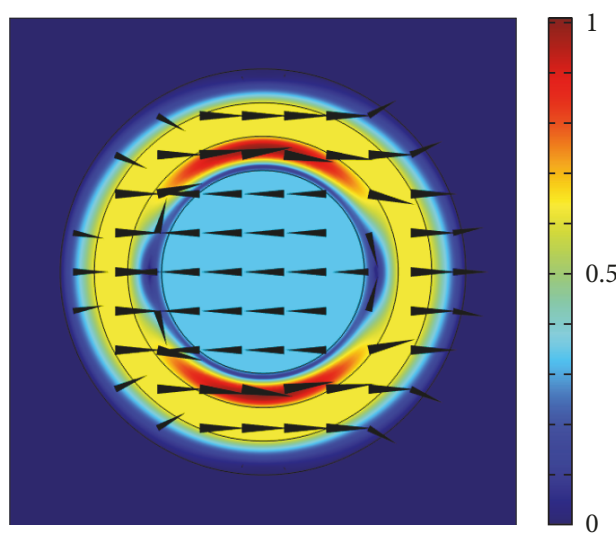

(c)

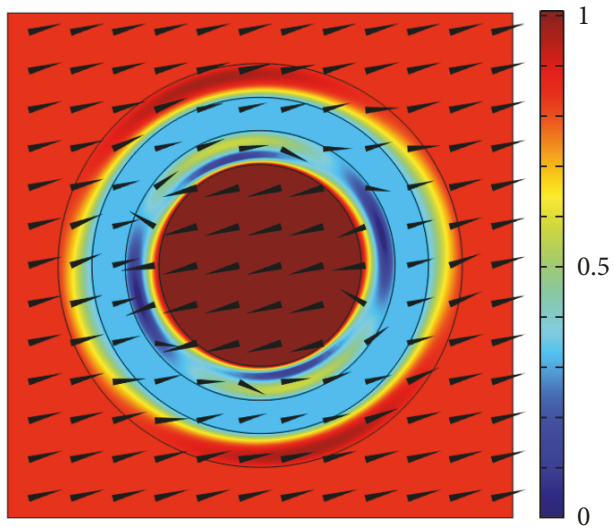

(b)

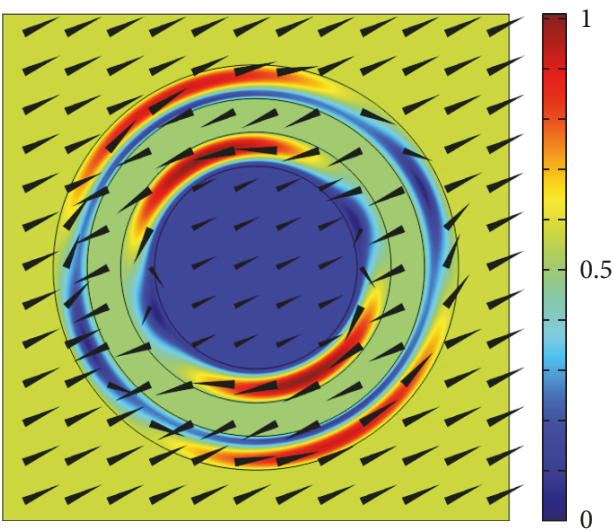

(d)

Figure 5: Vibration modes at the lower edge (a) and upper edge (b) of the first band gap; vibration modes at the lower edge (c) and upper edge (d) of the second band gap of the dual-oscillator acoustic metamaterial system. The color bar represents the normalized displacement.

outermost steel columns and the energy is concentrated on the central steel column and the matrix. Figures 7 (c) and $7(d)$ show the edge vibration modes of the second band gap. In this band gap, at lower edge, the central steel column vibrates opposite the intermediate and outermost steel columns while the matrix does not move, and the energy is distributed in the three steel columns; at the upper edge, the central steel column and the matrix vibrate opposite the intermediate and the outermost columns, and the energy is concentrated on the intermediate steel column and the matrix. Figures $7(\mathrm{e})$ and 7 (f) show the edges of the third band gap. In this band gap, at the lower edge, the intermediate layer vibrates opposite the outermost and center steel columns while the matrix does not move and the energy is mainly concentrated on the outermost and intermediate steel column; at the upper edge, the matrix and the intermediate steel columns vibrate opposite the outermost and center steel columns and the energy is concentrated on the outermost steel column.

Similar to the single-oscillator and dual-oscillator systems, the vibration modes of the triple-oscillator system are characterized by each layer of steel columns moving as an independent whole, where the Si-rubber layers connect the matrix and each layer of steel columns like springs. There is one main commonality in the vibration characteristics of these three acoustic metamaterial systems: the vibration modes at the lower edges of the band gaps are controlled only by the scatterer, while the matrix does not move; the matrix is involved only in the vibration at the upper edges. The lower edges of the band gaps of the triple-oscillator system can also be obtained by calculating the eigenfrequencies of a simplified mass-spring model which includes three mass blocks and three springs. The values we calculated for the lower edges of the first, second, and third band gap are $844 \mathrm{~Hz}, 2691 \mathrm{~Hz}$, and $4150 \mathrm{~Hz}$, respectively.

Figure 6(b) shows the transmission characteristic curve of the triple-oscillator acoustic metamaterial in the $\Gamma X$ direction, where the blue solid line and green dashed line represent the $p$-wave and $s$-wave, respectively. The maximum values of $p$-wave and $s$-wave vibration damping appeared at $1030 \mathrm{~Hz}, 2460 \mathrm{~Hz}, 3780 \mathrm{~Hz}$, and $6130 \mathrm{~Hz}$. Similar to the single-oscillator and dual-oscillator acoustic metamaterial systems, the maximum vibration attenuations in the tripleoscillator system corresponded to the original frequencies of the band gaps. Figures $6(\mathrm{c})-6(\mathrm{e})$ show the normalized effective mass density $\rho^{\text {eff }} / \rho_{0}$, effective bulk modulus $\kappa^{\text {eff }} / \kappa_{0}$, and effective shear modulus $\mu^{\text {eff }} / \mu_{0}$. In Figure 6(c), $\rho^{\text {eff }} / \rho_{0}$ becomes negative at $1029 \mathrm{~Hz}-1609 \mathrm{~Hz}, 2469 \mathrm{~Hz}-2949 \mathrm{~Hz}$, $3789 \mathrm{~Hz}-4149 \mathrm{~Hz}$, and $6209 \mathrm{~Hz}-6249 \mathrm{~Hz}$. The negative $\rho^{\text {eff }} / \rho_{0}$ 

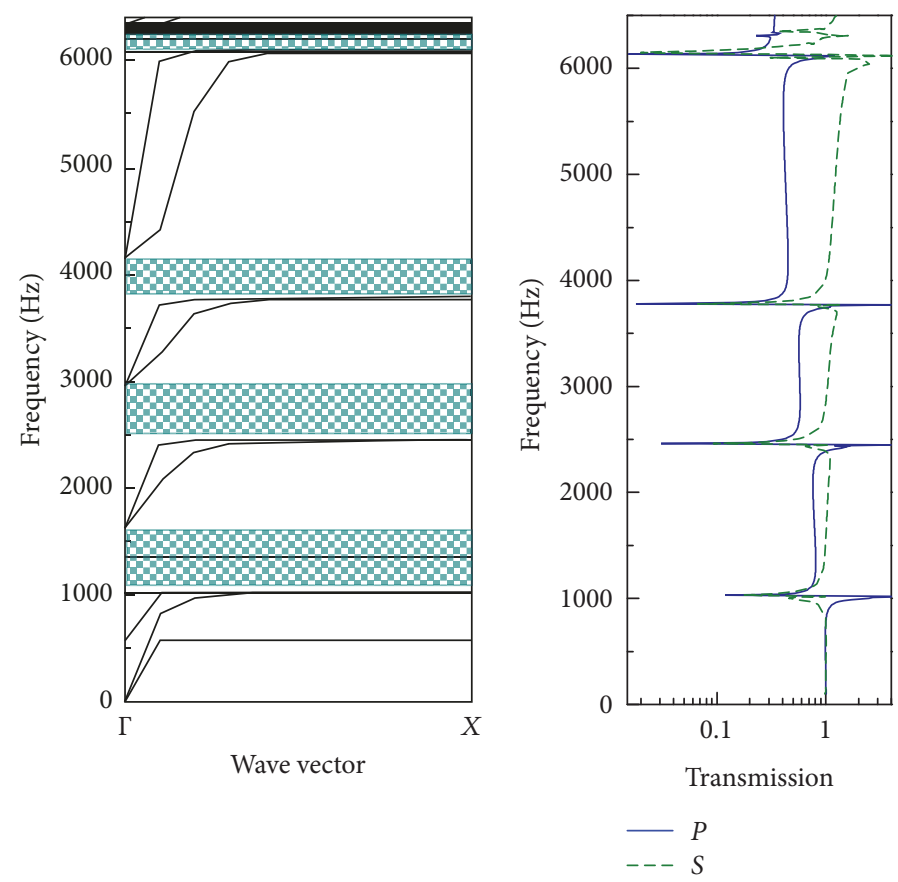

(a)

(b)

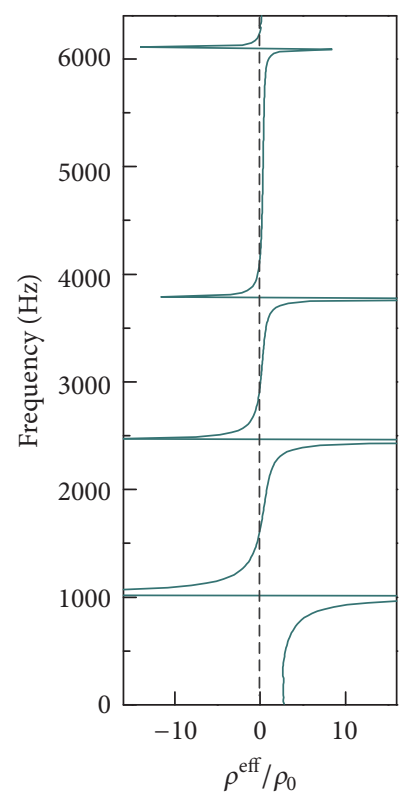

(c)

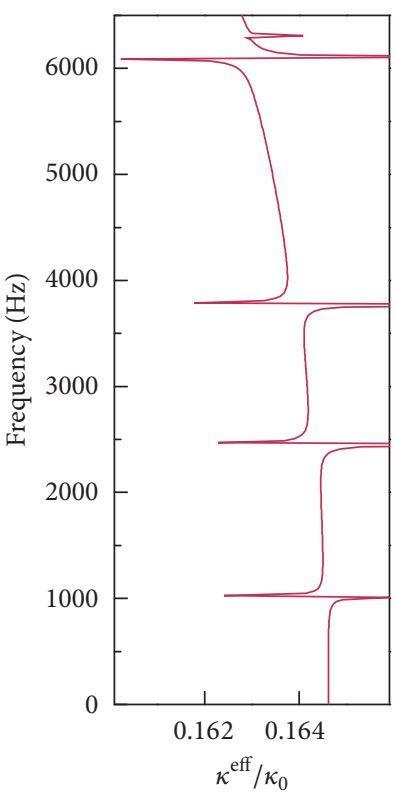

(d)

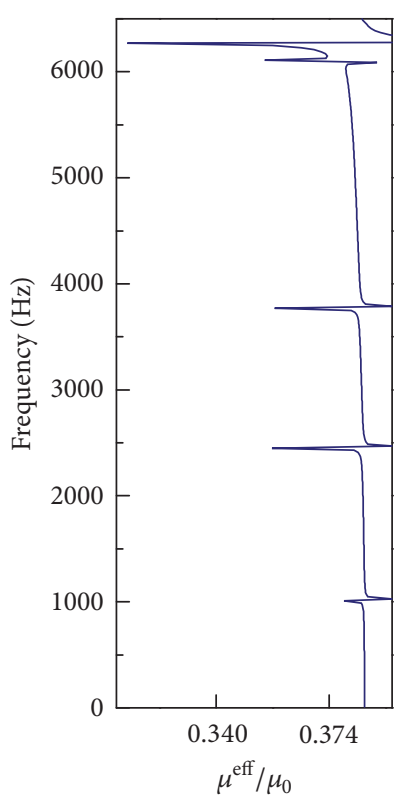

(e)

FIGURE 6: Band structure of triple-oscillator acoustic metamaterial in the $\Gamma X$ direction (a); transmission characteristic curve in the $\Gamma X$ direction (b); normalized effective mass density, effective bulk modulus, and effective shear modulus (c-e).

values correspond to the band gap, and positive-negative values change in a similar manner to those in the singleand dual-oscillator systems. In the $0 \mathrm{~Hz}-5000 \mathrm{~Hz}$ frequency range, $\kappa^{\mathrm{eff}} / \kappa_{0}$ and $\mu^{\mathrm{eff}} / \mu_{0}$ are positive; therefore, the tripleoscillator acoustic metamaterials are also single negative acoustic metamaterials. As evidenced by the observed vibration modes, the negative effective mass density was induced mainly by dipolar resonances of the scatterer (similarly to the other two systems).
3.4. Comparison of Three Acoustic Metamaterials. In the comparison of the three systems, a series of flat bands appears in all the band gaps, which is characteristic of local resonant acoustic metamaterials. As discussed above, the systems differed in their respective number of band gaps and their frequency ranges. The number of band gaps and their located frequencies change as the number of oscillators change, as shown in Table 3. Different with other resonance-coupling systems [42-44], the bandwidth of the original band gap 


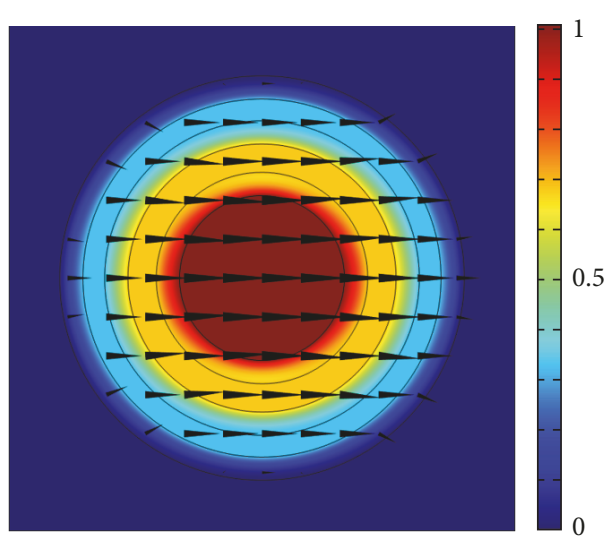

(a)

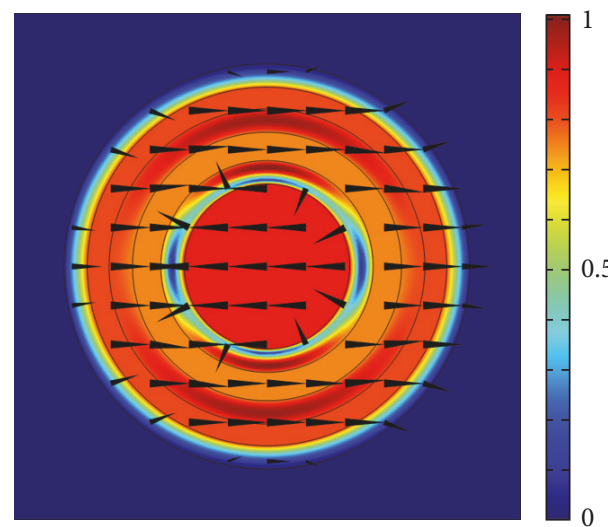

(c)

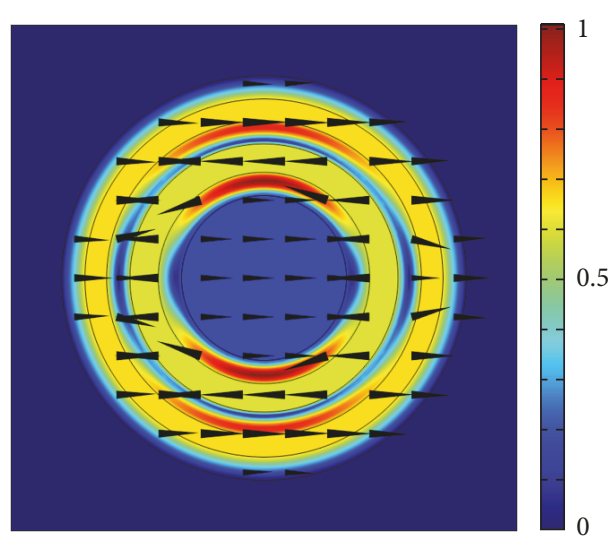

(e)

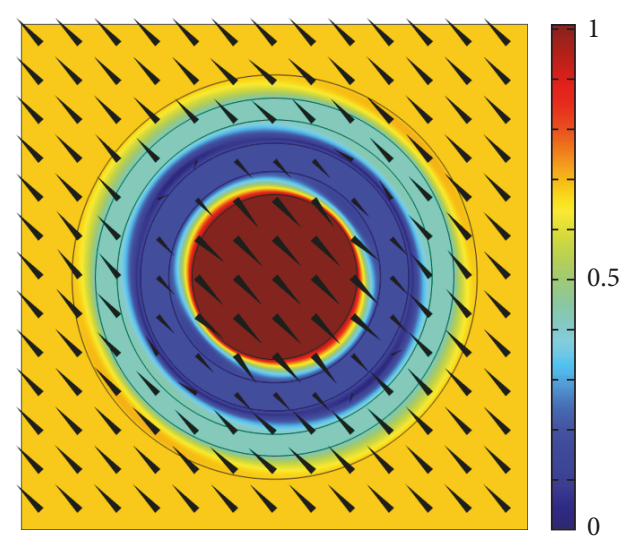

(b)

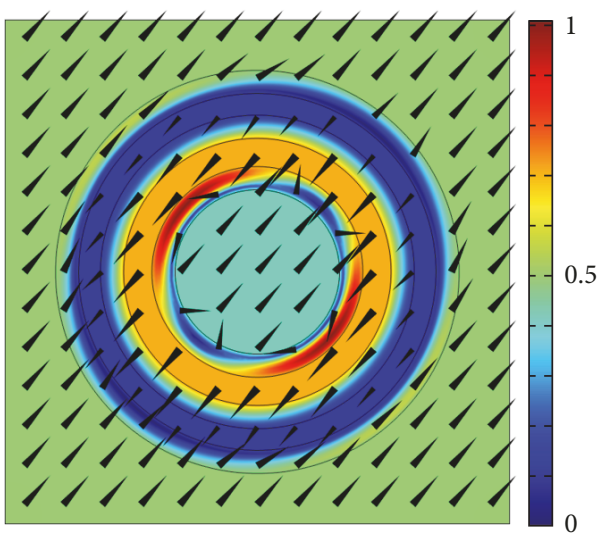

(d)

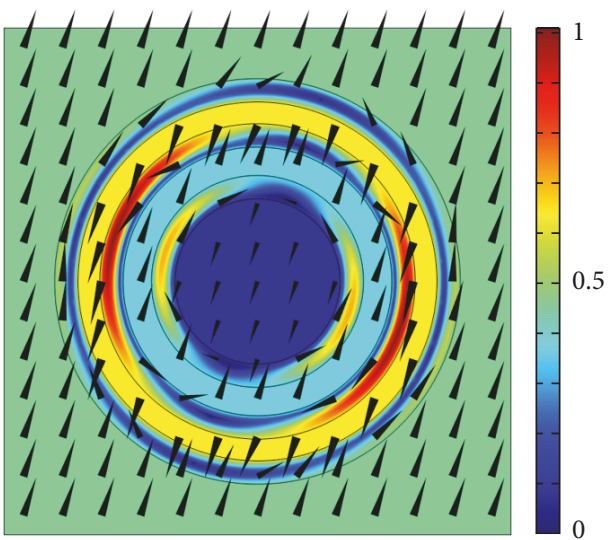

(f)

FIgURE 7: Vibration modes at lower edge (a) and upper edge (b) of the first band gap, at lower edge (c) and upper edge (d) of the second band gap, and at lower edge (e) and upper edge (f) of the third band gap in the triple-oscillator acoustic metamaterial system. The color bar represents the normalized displacement.

has not been significantly widened, but the number of band gaps in the system increased as the number of oscillators increased: specifically, each additional oscillator introduced a new band gap. The hierarchical design of the scatterer allows the opposite motion of the hard layers in the vibration modes of dual- and triple-oscillator metamaterials. That is to say, coupling appears in each part of the scatterer and as the number of oscillators increased, and more and more vibration modes emerge due to the coupling effect. As such, the number of band gaps increases as the number of oscillators increases due to a significant increase in the vibration freedoms.

It is also noted that the original frequency of the first band gap gradually increases as the number of oscillators increases. The reason can be identified by vibration mode information at edges of the first band gap and the results of (1). The vibration modes at the lower edge of the first band 
TABLE 3: Band gaps of single-, dual-, triple-oscillator systems.

\begin{tabular}{|c|c|c|c|c|}
\hline & Band gap edges $(\mathrm{Hz})$ & Single-oscillator & Dual-oscillator & Triple-oscillator \\
\hline \multirow{2}{*}{ The 1st band gap } & Lower & 846 & 946 & 1021 \\
\hline & Upper & 1542 & 1562 & 1621 \\
\hline \multirow{2}{*}{ The 2nd band gap } & Lower & 2571 & 2283 & 2452 \\
\hline & Upper & 2915 & 2835 & 2956 \\
\hline \multirow{2}{*}{ The 3rd band gap } & Lower & -- & 4247 & 3773 \\
\hline & Upper & -- & 4465 & 4125 \\
\hline \multirow{2}{*}{ The 4th band gap } & Lower & -- & -- & 6103 \\
\hline & Upper & -- & -- & 6257 \\
\hline
\end{tabular}

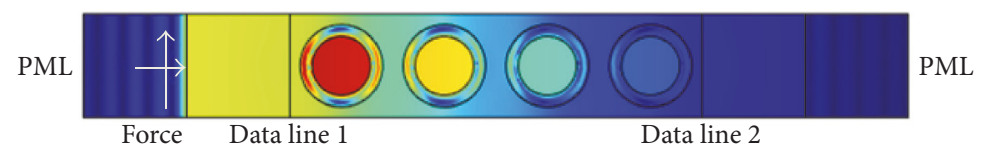

Figure 8: Numerical model for transmission computation. The color represents the displacement.

gap of all three systems are the same (as shown in Figures 3,5 , and 7), where the steel columns vibrate in the same direction. If the steel columns and Si-rubber between them in the dual- and triple-oscillator systems are considered as a whole, the vibration mode at the lower edge of the band gap in those systems can be simplified as a single-oscillator, massspring model-but because the dual-oscillator steel column is divided into two parts and the triple-oscillator steel column is divided into three parts, the vibration energy is concentrated in the central steel column so that the mass in the mass-spring simplified model decreases as the number of the oscillators increases. The effect of changes in spring stiffness coefficient is smaller compared to changes in mass, so (1) shows where the original frequency of the first band gap in the system increases as the number of oscillators increases.

Acoustic wave propagation in solids can be explained from the perspective of the effective medium parameters [8]. Existing research has confirmed that this analysis method is extremely effective $[3,36]$. In solids, the velocity of a longitudinal wave is determined by $\sqrt{(\kappa+\mu) / \rho}$ and the velocity of a shear wave is determined by $\sqrt{\mu / \rho}$, where $\rho$ is the mass density, $\kappa$ is the bulk modulus, and $\mu$ is the shear modulus. If $(\kappa+\mu) / \rho$ is negative, the longitudinal wave is an evanescent wave (i.e., one unable to propagate), and if one of $\mu$ or $\rho$ is negative, the shear wave is evanescent. For composite materials, wave propagation can be determined by calculating the effective medium parameters. All the single-, dual-, and triple-oscillator acoustic metamaterials achieve negative effective mass density within the band gap frequency range. The negative dynamic mass density in each system is induced by dipolar vibration at the lower edges of the band gaps. It is also found that bulk modulus and shear modulus are positive in the examined frequency ranges. All three systems are comprised of single negative acoustic metamaterials; thus, according to the theory of efficient medium parameters, longitudinal waves and shear waves in the frequency ranges of the band gaps are evanescent. This is the reason that vibration attenuation appears in the transmission characteristic curves of the three systems. As the number of oscillators increases, the relative movements between the oscillators generate coupling effect, increasing the dipolar resonance modes and creating negative effective mass density in more frequency ranges, thus increasing the number of band gaps.

\section{Conclusion}

By controlling the scatterer structure, a series of different 2D acoustic metamaterial systems are created: one with a single-oscillator, one with two oscillators, and one with three oscillators. The unique acoustic performance of each system is observed and analyzed using the finite element method and it was found that as the number of oscillators increases, the number of band gaps increases and the original frequency of the first band gap gradually increases. Then the physical mechanism of the band gap is clarified by examining the vibration modes and effective parameters in each system. In terms of the single-, dual-, and tripleoscillator models, all the dipolar resonances of the scatterer induce negative effective mass density, causing evanescent compression waves and shear waves which form band gaps. In addition, as the number of oscillators increase, there are relative movements between the oscillators that generated coupling effect, causing increase of dipolar resonance modes and making more negative effective mass density frequency ranges, further increasing the number of band gaps. The findings of this study provide valuable reference for designing acoustic metamaterials and acoustic devices in the future.

\section{Appendix}

\section{Transmission Calculation}

We established a numerical model based on FEM to illustrate the transmission properties of a $2 \mathrm{D}$ acoustic metamaterial system. The model is composed of a slab with four units along the $\Gamma X$ direction (Figure 8). Two Perfectly Matched Layers 
(PML) were added at its left and right sides to eliminate the effect of the reflective field. On the upper and lower edges, periodic boundary conditions were imposed to ensure infinite condition in this direction:

$$
\begin{aligned}
& u(x, y+a)=u(x, y) e^{i\left(k_{y} \cdot a\right)}, \\
& v(x, y+a)=v(x, y) e^{i\left(k_{y} \cdot a\right),}
\end{aligned}
$$

where $k_{y}$ are the components of the Bloch wave vector in the $y$ directions, respectively, and $a$ is the lattice constant, and $u$, $v$ are the components of the displacement in $x, y$ directions.

Prior to calculation, an external normal/tangential harmonic force was exerted on the left side to provide an input of longitudinal/transverse waves. The transmissions for the longitudinal or transverse waves were then obtained by calculating the ratio of integrating the horizontal or vertical displacements on data line 1 and data line 2 :

$$
\begin{aligned}
& T_{p}=\frac{\left.\int u \cdot d y\right|_{\text {line2 }}}{\left.\int u \cdot d y\right|_{\text {line1 }}}, \\
& T_{s}=\frac{\left.\int v \cdot d y\right|_{\text {line2 }}}{\left.\int v \cdot d y\right|_{\text {line1 }}} .
\end{aligned}
$$

\section{Conflicts of Interest}

The authors declare that they have no conflicts of interest.

\section{Acknowledgments}

The authors acknowledge project supported by the National Natural Science Foundation of China (Grant nos. 11202211 and 11602269), the Strategic Priority Research Program of the Chinese Academy of Sciences (Grant no. XDB22040301), and the Research Program of Beijing (Grant nos. Z161100002616034 and Z171100000817010).

\section{References}

[1] Y. F. Wang, Y. S. Wang, and L. Wang, "Two-dimensional ternary locally resonant phononic crystals with a comblike coating," Journal of Physics D: Applied Physics, vol. 47, Article ID 015502, 2014.

[2] T. Y. Chiang, L.-Y. Wu, C.-N. Tsai, and L. W. Chen, "A multilayered acoustic hyperlens with acoustic metamaterials," Applied Physics A, vol. 103, pp. 355-359, 2011.

[3] Y. Lai, Y. Wu, P. Sheng, and Z. Q. Zhang, "Hybrid elastic solids," Nature Materials, vol. 10, no. 8, pp. 620-624, 2011.

[4] Z. Liu, X. Zhang, Y. Mao et al., "Locally resonant sonic materials," Science, vol. 289, no. 5485, pp. 1734-1736, 2000.

[5] Z. Liu, C. T. Chan, and P. Sheng, "Analytic model of phononic crystals with local resonances," Physical Review B, vol. 71, no. 1, Article ID 014103, 2005.

[6] Z. Yang, J. Mei, M. Yang, N. H. Chan, and P. Sheng, "Membranetype acoustic metamaterial with negative dynamic mass," Physical Review Letters, vol. 101, no. 20, Article ID 204301, 2008.

[7] N. Fang, D. Xi, J. Xu et al., "Ultrasonic metamaterials with negative modulus," Nature Materials, vol. 5, no. 6, pp. 452-456, 2006.
[8] X. Zhou and G. Hu, "Analytic model of elastic metamaterials with local resonances," Physical Review B, vol. 79, Article ID 195109, 2009.

[9] Y. Wu, Y. Lai, and Z. Q. Zhang, "Effective medium theory for elastic metamaterials in two dimensions," Physical Review B, vol. 76, Article ID 205313, 2007.

[10] Y. Ding, Z. Liu, C. Qiu, and J. Shi, "Metamaterial with simultaneously negative bulk modulus and mass density," Physical Review Letters, vol. 99, no. 9, Article ID 093904, 2007.

[11] J. Li and C. Chan, "Double-negative acoustic metamaterial," Physical Review E, vol. 70, Article ID 055602, 2004.

[12] K. Deng, Y. Ding, Z. He, H. Zhao, J. Shi, and Z. Liu, “Theoretical study of subwavelength imaging by acoustic metamaterial slabs," Journal of Applied Physics, vol. 105, no. 12, p. 124909, 2009.

[13] M. B. Assouar and M. Oudich, "Enlargement of a locally resonant sonic band gap by using double-sides stubbed phononic plates," Applied Physics Letters, vol. 100, no. 12, Article ID 123506, 2012.

[14] Y. Xiao, B. R. MacE, J. Wen, and X. Wen, "Formation and coupling of band gaps in a locally resonant elastic system comprising a string with attached resonators," Physics Letters A, vol. 375, no. 12, pp. 1485-1491, 2011.

[15] P. Yeh, A. Yariv, and E. Maron, “Theory of bragg fiber," Journal of the Optical Society of America, vol. 68, no. 9, pp. 1196-1201, 1978.

[16] A. Kitagawa and J.-I. Sakai, "Bloch theorem in cylindrical coordinates and its application to a Bragg fiber," Physical Review A, vol. 80, no. 3, Article ID 033802, pp. 2962-2964, 2009.

[17] H. Larabi, Y. Pennec, B. Djafari-Rouhani, and J. O. Vasseur, "Multicoaxial cylindrical inclusions in locally resonant phononic crystals," Physical Review E, vol. 75, no. 6, Article ID 066601, 2007.

[18] D. Torrent and J. Sánchezdehesa, "Radial wave crystals: radially periodic structures from anisotropic metamaterials for engineering acoustic or electromagnetic waves," Physical Review Letters, vol. 103, Article ID 064301, 2009.

[19] Z. Xu, F. Wu, and Z. Guo, "Low frequency phononic band structures in two-dimensional arc-shaped phononic crystals," Physics Letters A, vol. 376, pp. 2256-2263, 2012.

[20] X. Zhou and C. Chen, "Tuning the locally resonant phononic band structures of two-dimensional periodic electroactive composites," Physica B: Condensed Matter, vol. 431, pp. 23-31, 2013.

[21] X. Zhang, Y. Liu, F. Wu, and Z. Liu, "Large two-dimensional band gaps in three-component phononic crystals," Physics Letters A, vol. 317, no. 1-2, pp. 144-149, 2003.

[22] G. Wang, L. Shao, Y. Liu, and J. Wen, Chin. Phys, vol. 15, pp. 1843-1848, 2006.

[23] Y. Wu, Y. Lai, and Z. Zhang, "Effective medium theory for elastic metamaterials in two dimensions," Physical Review B, vol. 76, Article ID 205313, 2007.

[24] P. Sheng, J. Mei, Z. Liu, and W. Wen, "Dynamic mass density and acoustic metamaterials," Physica B: Condensed Matter, vol. 394, no. 2, pp. 256-261, 2007.

[25] B. Popa and S. A. Cummer, "Design and characterization of broadband acoustic composite metamaterials," Physical Review $B$, vol. 80, no. 17, Article ID 174303, 2009.

[26] L. Zigoneanu, B. Popa, A. F. Starr, and S. A. Cummer, "Design and measurements of a broadband two-dimensional acoustic metamaterial with anisotropic effective mass density," Journal of Applied Physics, vol. 109, no. 5, p. 054906, 2011.

[27] R. E. Camley, B. Djafari-Rouhani, L. Dobrzynski, and A. A. Maradudin, "Sagittal elastic waves in infinite and semi-infinite superlattices," Physical Review B, vol. 28, pp. 1711-1720, 1983. 
[28] Z. Liu, C. T. Chan, P. Sheng, A. L. Goertzen, and J. H. Page, "Elastic wave scattering by periodic structures of spherical objects: theory and experiment," Physical Review B, vol. 62, no. 4, pp. 2446-2457, 2000.

[29] I. E. Psarobas, N. Stefanou, and A. Modinos, "Scattering of elastic waves by periodic arrays of spherical bodies," Physical Review B, vol. 62, no. 1, pp. 278-291, 2000.

[30] Y. Tanaka, Y. Tomoyasu, and S.-I. Tamura, "Band structure of acoustic waves in phononic lattices: two-dimensional composites with large acoustic mismatch," Physical Review B, vol. 62 , no. 11, pp. 7387-7392, 2000.

[31] J. S. Jensen, "Phononic band gaps and vibrations in one- and two-dimensional mass-spring structures," Journal of Sound and Vibration, vol. 266, no. 5, pp. 1053-1078, 2003.

[32] G. Wang, J. H. Wen, Y. Z. Liu, and X. S. Wen, "Lumped-mass method for the study of band structure in two-dimensional phononic crystals," Physical Review B, vol. 69, no. 18, Article ID 184302, 2004.

[33] D. Krattiger and M. I. Hussein, "Bloch mode synthesis: ultrafast methodology for elastic band-structure calculations," Physical Review E, vol. 90, no. 6, Article ID 069904, 2014.

[34] J. Wen, H. Zhao, L. Lv, B. Yuan, G. Wang, and X. Wen, "Effects of locally resonant modes on underwater sound absorption in viscoelastic materials," The Journal of the Acoustical Society of America, vol. 130, no. 3, pp. 1201-1208, 2011.

[35] I. A. Veres, T. Berer, and O. Matsuda, "Complex band structures of two dimensional phononic crystals: analysis by the finite element method," Journal of Applied Physics, vol. 114, no. 8, Article ID 083519, 2013.

[36] X. N. Liu, G. K. Hu, G. L. Huang, and C. T. Sun, "An elastic metamaterial with simultaneously negative mass density and bulk modulus," Applied Physics Letters, vol. 98, no. 25, p. 251907, 2011.

[37] M. Hirsekorn, "Small-size sonic crystals with strong attenuation bands in the audible frequency range," Applied Physics Letters, vol. 84, no. 17, pp. 3364-3366, 2004.

[38] Y. F. Wang, Y. S. Wang, and V. Laude, "Wave propagation in two-dimensional viscoelastic metamaterials," Physical Review B, vol. 92, Article ID 104110, 2015.

[39] Y. F. Wang, V. Laude, and Y. S. Wang, "Coupling of evanescent and propagating guided modes in locally resonant phononic crystals," Journal of Physics D: Applied Physics, vol. 47, no. 47, Article ID 475502, 2014.

[40] P. G. Domadiya, E. Manconi, M. Vanali, L. V. Andersen, and A. Ricci, "Numerical and experimental investigation of stop-bands in finite and infinite periodic one-dimensional structures," Journal of Vibration and Control, vol. 22, no. 4, pp. 920-931, 2016.

[41] C. Goffaux, J. Sánchez-Dehesa, A. L. Yeyati et al., "Evidence of fano-like interference phenomena in locally resonant materials," Physical Review Letters, vol. 88, no. 22, Article ID 225502, 2002.

[42] M. Chen, D. Meng, H. Zhang, H. Jiang, and Y. Wang, "Resonance-coupling effect on broad band gap formation in locally resonant sonic metamaterials," Wave Motion, vol. 63, pp. 111-119, 2016.

[43] M. Badreddine Assouar, J.-H. Sun, F.-S. Lin, and J.-C. Hsu, "Hybrid phononic crystal plates for lowering and widening acoustic band gaps," Ultrasonics, vol. 54, no. 8, pp. 2159-2164, 2014.

[44] Y. Cheng, J. Y. Xu, and X. J. Liu, "Broad forbidden bands in parallel-coupled locally resonant ultrasonic metamaterials," Applied Physics Letters, vol. 92, no. 5, p. 051913, 2008. 


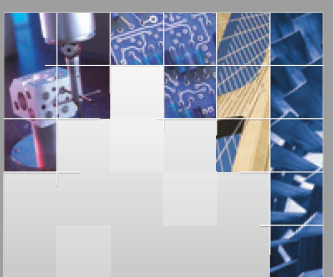

\section{Enfincering}
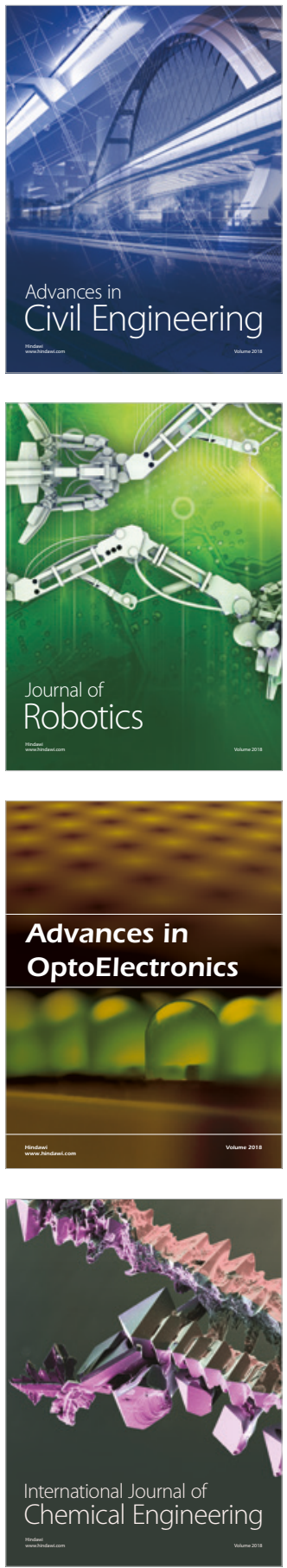

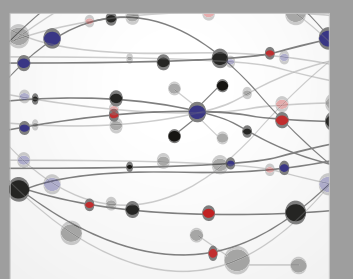

\section{Rotating \\ Machinery}

The Scientific World Journal

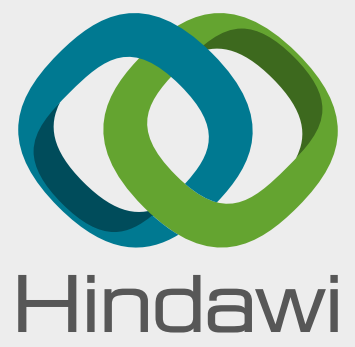

Submit your manuscripts at

www.hindawi.com
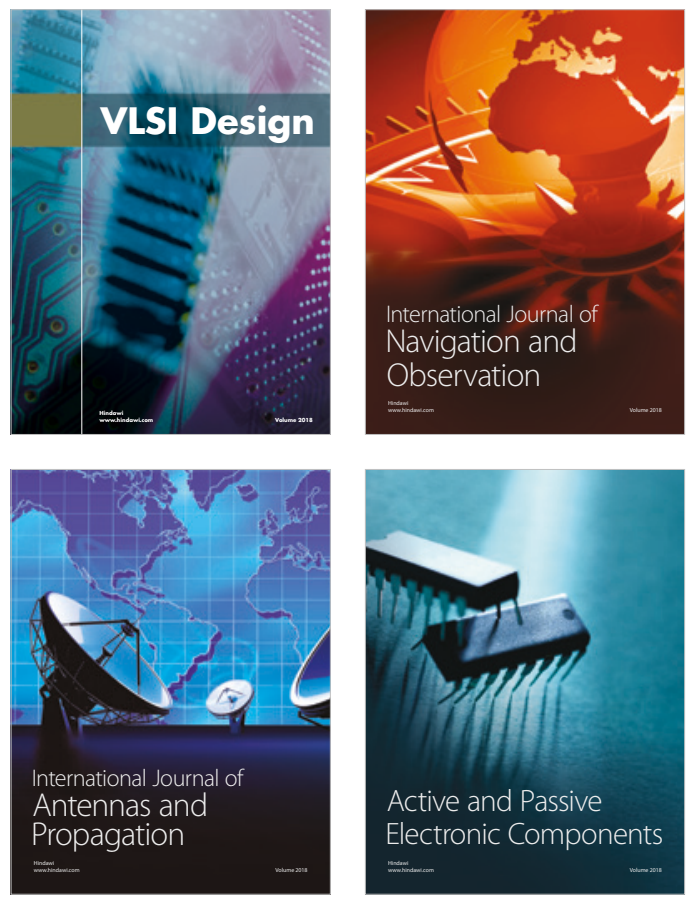
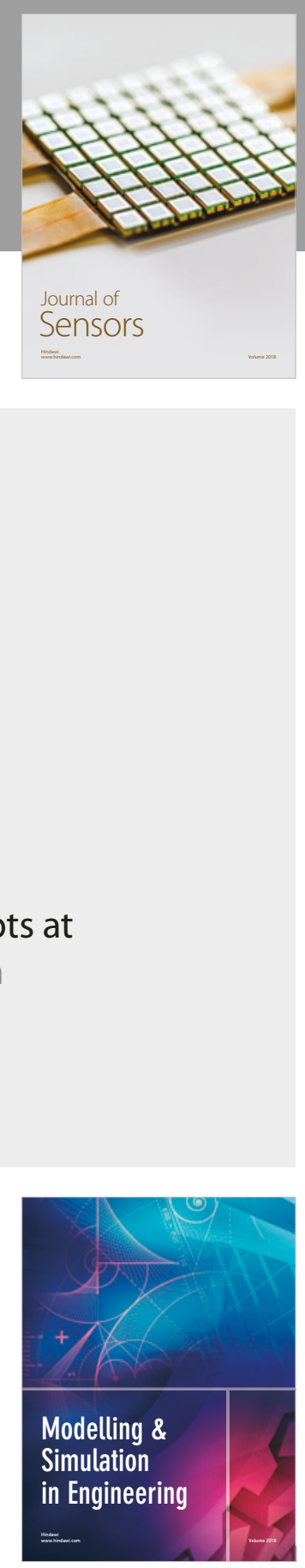

\section{Advances \\ Multimedia}
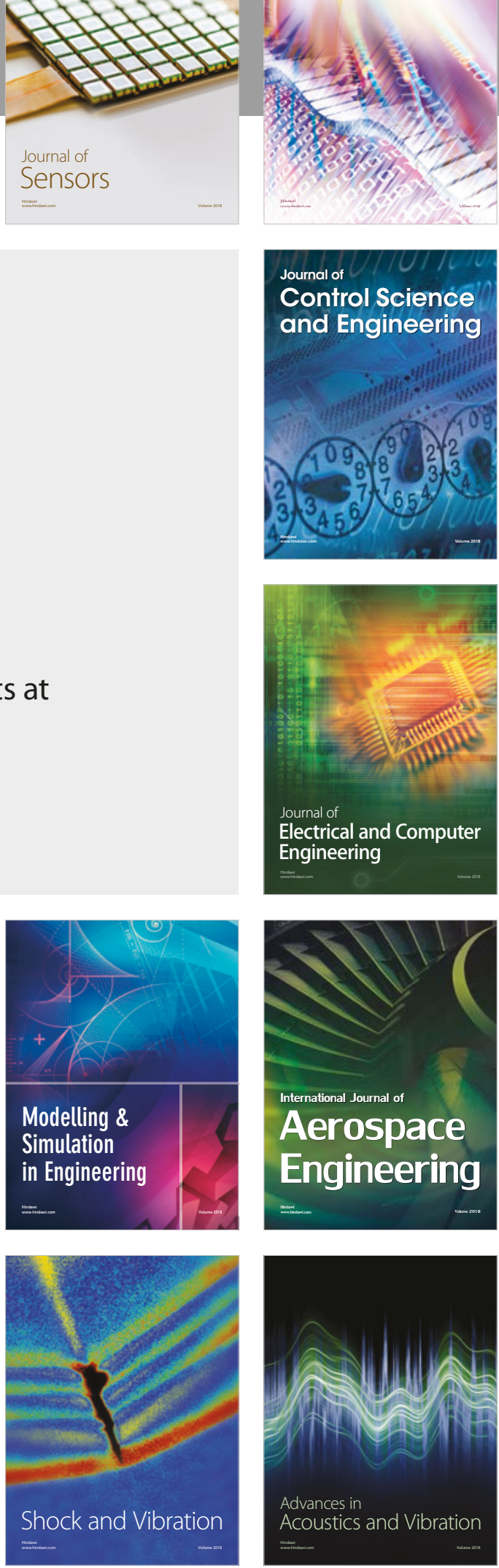